\title{
GENERIC PARAMETERS GOVERNING THE WETTABILITY CHARACTERISTICS OF LASER-MODIFIED NYLON 6,6
}

\author{
Paper (M1204) \\ David Waugh and Jonathan Lawrence \\ School of Engineering, University of Lincoln, Brayford Pool, Lincoln, Lincolnshire, LN6 7TS, UK.
}

\begin{abstract}
Wettability is an interesting subject which spans any discipline that takes into account any form of adhesion. As such, it is imperative to identify generic parameters that govern the wetting nature of materials. This paper details the use of $\mathrm{CO}_{2}$ and $\mathrm{KrF}$ excimer lasers to modify the surface of nylon 6,6 in order to modulate the wettability characteristics. White light interferometery allowed one to establish that the surface roughness $(\mathrm{Ra})$ was dramatically increased by up to $4.5 \mu \mathrm{m}$ in comparison to the as-received sample. Using a sessile drop device it was determined for all samples that the polar component had a strong inverse relationship with the contact angle, $\theta$. For the patterned samples an increase in $\theta$ indicated the likely formation of a mixed-state wetting regime and highlighted the significant influence of surface topography on $\theta$. X-ray photoelectron spectroscopy determined that the surface oxygen content had increased by up to $5.7 \%$ at. for all samples. Due to the highly modulated nature of $\theta$ over all samples it was concluded that surface oxygen content was not a dominant parameter; whereas the polar component and surface pattern were the most dominant parameters governing the wettability characteristics of the laser-surface-modified nylon 6,6.
\end{abstract}

\section{Introduction}

When considering the subject of hydrophilic surfaces and the wettability of materials the term 'wetting' is a fundamental phenomena that should always initially be taken into account. The understanding of the wetting of a surface by liquids leading to the spreading of those liquids over the surface can be seen to be a crucial factor that is incorporated within surface chemistry. The process of wetting has been recognized and researched for many years by many scientists; however, like many subjects within the scientific community the process is still not fully understood $(1,2)$. Even though this process is still not fully understood, many breakthroughs have occurred in this subject area allowing for a more thorough understanding between the interactions of surfaces, interfaces and the biological environment. Leading on, various laser-based techniques for altering the wettability characteristics of numerous materials have been developed $(2,3)$. It should also be noted here that various other methods have been developed such as the research carried out by Dumitrascu et al. (4) highlighting the possibility of using a pulsed dielectric barrier discharge (DBD) to modify the roughness of surfaces for various materials.

Previous work (5-7) has demonstrated the potential of using $\mathrm{CO}_{2}$ and excimer lasers to modify the surfaces of polymeric materials. On account of this, this paper details a study into the surface modification of nylon 6,6 using $\mathrm{CO}_{2}$ and excimer lasers in the endeavour to determine generic parameters governing the lasermodified wettability characteristics.

\section{Experimental Technique}

\section{Nylon 6,6 Material}

The nylon 6,6 $\left(\mathrm{T}_{\mathrm{m}}: 255^{\circ}, \rho: 1.3 \mathrm{gcm}^{-3}\right)$ was sourced in $100 \mathrm{~mm}^{2}$ sheets with a thickness of $5 \mathrm{~mm}$ (Goodfellow Cambridge, Ltd). To obtain a conveniently sized sample for experimentation the as-received nylon sheet was cut into $20 \mathrm{~mm}$ diameter discs using a $1 \mathrm{~kW}$ continuous wave (cw) $\mathrm{CO}_{2}$ laser (Everlase $\mathrm{S} 48$; Coherent, Ltd).

\section{$\mathrm{CO}_{2}$ Laser-Induced Patterning}

In order to generate the required marking pattern with the Synrad $10 \mathrm{~W} \mathrm{CO}_{2}$ laser system, Synrad Winmark software version 2.1.0, build 3468 was used. The software was capable of using images saved as .dxf files which can be produced by using CAD programs such as, in this case, Licom AutoCaM. The nylon 6,6 samples were placed into the laser system onto a stage in which they were held in place using a bracket with a $20.5 \mathrm{~mm}$ diameter hole cut into the centre of the bracket. The surface of the sample was set to be 250 $\mathrm{mm}$ away from the output facet of the laser system to obtain focus and the system employed a galvanometer scanner to scan the $95 \mu \mathrm{m}$ spot size beam directly across the stationary target material. It should be noted that the target material and laser system was held in a 
laser safety cabinet in which the ambient gas was air and an extraction system was used to remove any fumes produced during laser processing.

There were four patterns induced onto the surfaces of the nylon 6,6 samples; were trenches with $50 \mu \mathrm{m}$ spacing (CT50), hatch with $50 \mu \mathrm{m}$ spacing (CH50), trenches with $100 \mu \mathrm{m}$ spacing (CT50) and hatch with $100 \mu \mathrm{m}$ spacing (CH100). In addition, an as-received control sample was used (A1). For each of the irradiated patterns the laser power was set to $70 \%$ (7 W) operating at $600 \mathrm{mms}^{-1}$.

\section{$\mathrm{CO}_{2}$ Laser Whole Area Irradiative Processing}

A cw $100 \mathrm{~W} \mathrm{CO}_{2}$ laser (DLC; Spectron, Ltd) was used to scan a $5 \mathrm{~mm}$ diameter beam across the target sample with one pass in order to irradiate the test area with an irradiance of $510 \mathrm{Wcm}^{2}$. By using a galvanometer, scanning speeds of $150,100,75,50,25$ and $20 \mathrm{mms}^{-1}$ were employed to irradiate six samples with effective fluences of 16.84 (samples CWA17), 25.51 (sample CWA26), 34.18 ( sample CWA34), 51.02 (sample CWA51), 102.04 (sample CWA102) and 127.55 (sample CWA128) $\mathrm{Jcm}^{-2}$, respectively. As with the laser-induced patterning experimentation the samples were held in place on a z-variable stage with the whole experiment being carried out in a laser safety cabinet with the ambient gas being air.

\section{KrF Excimer Laser-Induced Patterning}

For the patterned experiments the repetition rate was kept constant at $25 \mathrm{~Hz}$, with a number of 10 pulses per site and used Aerotech $\mathrm{CNC}$ programming to induce the required pattern. A constant laser energy of $80 \pm 7$ $\mathrm{mJ}$ was used with the attenuator set to $0.3(30 \%)$ giving a measured energy at the target sample of $23.67 \pm 2.5 \mathrm{~mJ}$, resulting in a fluence of $858 \pm 91$ $\mathrm{mJ} / \mathrm{cm}^{2}$. In order to induce the intended pattern a projection imaging system was implemented with a focusing lens of x10 demagnification. The patterns induced using this technique were $50 \mu \mathrm{m}$ trench (ET50), $100 \mu \mathrm{m}$ trench (ET100), $50 \mu \mathrm{m}$ hatch (EH50) and $100 \mu \mathrm{m}$ (EH100). Two non-contact masks were used for both dimensioned patterns which included a brass mask with six $1 \mathrm{~mm}$ diameter holes spaced by 2 $\mathrm{mm}$, centre to centre, for the $100 \mu \mathrm{m}$ dimensions and a SS316 foil (Laser Micromachining Ltd., UK) with five $0.5 \mathrm{~mm}$ diameter holes spaced by $1.5 \mathrm{~mm}$, 'centre to centre', for the $50 \mu \mathrm{m}$ dimensions. To keep the constant 10 pulses per site it should also be noted here that scanning velocities of $0.125 \mathrm{mms}^{-1}$ and $0.25 \mathrm{mms}^{-1}$ were used for the $50 \mu \mathrm{m}$ and $100 \mu \mathrm{m}$ dimensioned patterns, respectively.
No processing gases were used throughout the experimentation and all laser processing was carried out in an enclosure in which the ambient gas was air. Also, for all laser processing no homogonizer was implemented meaning that the raw beam was used which would have given rise to energy spikes pulse to pulse, having some possible effect on the incident laser fluence and laser material processing.

\section{KrF Excimer Laser Whole Area Irradiative Processing}

For the whole area processing with an excimer laser the raw $23 \times 12 \mathrm{~mm}^{2}$ beam was used to irradiate a large section of each sample at a time. In order to hold the sample normal to the beam a bracket on the optical train was used. For the large area processing experiments 6 samples where studied; these being 100 pulses at $100 \mathrm{~mJ}$ (EWA100), 100 pulses at $150 \mathrm{~mJ}$ (EWA150), 100 pulses at $200 \mathrm{~mJ}$ (EWA200), 100 pulses at $250 \mathrm{~mJ}$ (EWA250), 500 pulses at $250 \mathrm{~mJ}$ (EWA250_500) and 1000 pulses at $250 \mathrm{~mJ}$ (EWA250_1000). This gave fluences of $36 \pm 3,54 \pm 5$, $72 \pm 8$ and $\overline{9} 1 \pm 10 \mathrm{mJcm}^{-2}$, respectively for the different energies used. Throughout the whole area excimer experiments the repetition rate was kept constant at 25 $\mathrm{Hz}$ and Aerotech $\mathrm{CNC}$ programming ensured that the correct number of pulses was applied to each sample.

\section{Topography, Wettability Characteristics and Surface Chemistry Analysis}

After laser irradiation the nylon 6,6 samples were analysed using a number of techniques. The surface profiles were determined using a white light interferometer (WLI) (NewView 500; Zygo, Ltd) with MetroPro and TalyMap Gold Software. The WLI was set-up using a $\times 10$ Mirau lens with a zoom of $\times 0.5$ and working distance of $7.6 \mathrm{~mm}$. This system also allowed $\mathrm{Sa}$ and $\mathrm{Ra}$ roughness parameters to be determined for each sample.

In accordance with the procedure detailed by Rance (8) the samples were ultrasonically cleaned in isoproponal (Fisher Scientific Ltd.) for 3 minutes at room temperature before using a sessile drop device to determine various wettability characteristics. This was to allow for a relatively clean surface prior to any $\theta$ measurements being taken. To ensure that the sample surfaces were dry a specimen dryer (Metaserv, Ltd.) was employed to blow ambient air across the samples. A sessile drop device (OCA20; Dataphysics Instruments, $\mathrm{GmbH}$ ) was used with relevant software (SCA20; Dataphysics Intrsuments, $\mathrm{GmbH}$ ) to allow the recent advancing and receding $\theta$ for triply distilled water and the recent advancing angle for diiodomethane to be determined for each sample. 
Thereafter the advancing $\theta$ for the two liquids were used by the software to draw an OWRK plot to determine the surface energy of the samples. For the two reference liquids the SCA20 software used the Ström et al technique (triply distilled water SFT(total:72.80), SFT(D:21.80), SFT(P:51.00); diiodomethane - SFT(total:50.80), SFT(D:50.80), SFT(P:0.00)) to calculate the surface energy of the material. It should be noted here that ten $\theta$, using two droplets in each instance, were recorded to achieve a mean $\theta$ for each liquid and surface.

All samples were analysed using x-ray photoelectron spectroscopy (XPS). This allowed any surface modifications in terms of surface oxygen content due to the laser irradiation to be revealed. XPS measurements were performed on a photoelectron spectrometer (Axis Ultra DLD; Kratos Analytical Ltd., UK) employing monochromatic aluminium k-alpha radiation source, operating at $120 \mathrm{~W}$ power and an associated photon energy of $1486.6 \mathrm{eV}$. To test the reproducability of the surface, two sections of each sample were analysed; the analysis area on each sample was $700 \times 300$ microns. The spectrometer was run in its Hybrid mode and spectra were acquired at pass energies of $20 \mathrm{eV}$ (for the high resolution scans) and $160 \mathrm{eV}$ for the survey scans. All data was analysed through CasaXPS (v2.3.14) analysis software using sensitivity factors supplied by the instrument manufacturer.

\section{Results and Discussion}

\section{Topography $-\mathrm{CO}_{2}$ and $\mathrm{KrF}$ Excimer Laser- Induced Patterning}

A full detailed account of the topography modification for the $\mathrm{CO}_{2}$ and $\mathrm{KrF}$ Excimer Laser-induced patterned samples can be found in (5) and (6), respectively.

It was deduced that the $\mathrm{CO}_{2}$ laser-induced patterned samples had considerably rougher surfaces with the largest peak heights being of the order of $2 \mu \mathrm{m}$ in contrast to the as-received sample which had peaks heights of up to $0.2 \mu \mathrm{m}$. On account of the increase in peak heights over the $\mathrm{CO}_{2}$ laser-patterned samples the surface roughness (see Table 1) increased considerably with the largest $\mathrm{Sa}$ of $0.4 \mu \mathrm{m}$ being achieved with the $50 \mu \mathrm{m}$ hatch sample (CH50) and largest Ra of $0.2 \mu \mathrm{m}$ for the $100 \mu \mathrm{m}$ trench sample (CT100). It is given in Table 1 that the patterned samples with scan dimensions of $50 \mu \mathrm{m}$ (samples CT50 and CH50) had larger $\mathrm{Sa}$ roughness values when compared to the samples patterned with $100 \mu \mathrm{m}$ scan dimensions (samples CT100 and CH100). This can be attributed to the fact that the $50 \mu \mathrm{m}$ scan dimensions irradiated more of the sample giving rise to an increase of mass being melted and re-solidified. Also, it can be seen from the Table 1 that the roughness for the hatch patterns had decreased in comparison to the trench patterns. This can be explained by the laser re-melting sections of the nylon 6,6 surface owed to the scanning process of the system. By re-melting these sections the material could then have re-solified into a smoother surface topography.

It was evident that the $50 \mu \mathrm{m}$ patterns (CT50 and CH50) had the least periodicity, which can be attributed to the fact that the beam spot size was $95 \mu \mathrm{m}$ and consequently allowed the scans to overlap and thus eliminate the natural periodicity of the original scanned pattern. This could also allow one to explain the surface Sa roughness increase as seen in Table 1 as the scanned $50 \mu \mathrm{m}$ dimensioned patterns (samples CT50 and $\mathrm{CH} 50$ ) had up to a three times larger $\mathrm{Sa}$ value compared to the $100 \mu \mathrm{m}$ scan dimensioned nylon 6,6 samples (samples CT100 and CH100). That is on account of significantly more melting and resolidifcation taking place, on account of the overlapping, a rougher surface could have resulted.

For the excimer laser-induced patterned samples the topography of the nylon 6,6 had been modified significantly when compared to the as-received sample. For the $50 \mu \mathrm{m}$ dimensioned patterns (ET50 and EH50) trench depths of around $1 \mu \mathrm{m}$ was obtained from the employed laser processing parameters. These constant laser processing parameters also gave rise to trench depths of roughly 4 to $5 \mu \mathrm{m}$ for the $100 \mu \mathrm{m}$ dimensioned patterned samples (ET100 and EH100). What is more, on account of the accuracy and precision of the excimer laser system implemented, the trenches produced in the nylon 6,6 materials were highly periodic.

Table 2 gives the surface roughness values for each of the $\mathrm{KrF}$ excimer laser-induced patterned samples and shows that the surface roughness had dramatically increased for the excimer laser patterned samples with the largest increases being seen for the $100 \mu \mathrm{m}$ dimensioned patterns (ET100 and EH100) which had an Sa of approximately $1.5 \mu \mathrm{m}$. This can be seen to be on account of the fact that the $100 \mu \mathrm{m}$ dimensioned patterned samples gave rise to deeper trenches in comparison to the $50 \mu \mathrm{m}$ dimensioned samples.

\section{Topography $-\mathrm{CO}_{2}$ and KrF Excimer Laser Whole Area Processing}

A full detailed account of the topography modification for the $\mathrm{CO}_{2}$ and $\mathrm{KrF}$ Excimer Laser-induced patterned samples can be found in (5) and (6), respectively. 
The $\mathrm{CO}_{2}$ laser whole area irradiative processing of the nylon 6,6 gave rise to a significantly modified surface, especially with those samples which had been irradiated with large fluences (samples CWA102 and CWA128). This can be accounted for by more melting taking place arising from the significantly larger temperature rise owed to the large irradiances incident on the nylon 6,6 surfaces.

There was further evidence of considerable melting on account of one being able to identify craters left from evolved gases breaking at the surface. This is especially apparent for sample CWA102 and sample CWA128 which had larger incident fluences of 102 and $128 \mathrm{Jcm}^{-2}$. It should also be noted here that as the fluence used for samples CWA17 and CWA26 appeared experimentally to be close to that of the threshold, qualitatively there did not appear to be significant difference between the topographies observed for them and that of the as-received sample.

Through WLI it was found that the $\mathrm{KrF}$ excimer laser whole area irradiative processed samples appeared to have a similar topography to that of the as-received sample. This can be attributed to the fact that the whole sample was irradiated meaning that any ablation taking place would remove a somewhat uniform layer from the surface of the nylon 6,6. On the other hand, especially for the samples with low incident fluences, it may be possible that the material did not ablate on account of the threshold fluence not being achieved.

Table 1 - Results summary for samples showing roughness parameters, surface oxygen content and wettability characteristics following $\mathrm{CO} 2$ laser processing of nylon 6,6.

\begin{tabular}{|c|c|c|c|c|c|c|c|}
\hline $\begin{array}{c}\text { Sample } \\
\text { ID }\end{array}$ & $\mathrm{Sa}$ & $\mathrm{Ra}$ & $\begin{array}{c}\text { Polar } \\
\text { Component, } \gamma^{\mathrm{P}} \\
\left(\mathrm{mJm}^{-2}\right)\end{array}$ & $\begin{array}{c}\text { Dispersive } \\
\text { Component, } \gamma^{\mathrm{D}} \\
\left(\mathrm{mJm}^{-2}\right)\end{array}$ & $\begin{array}{c}\text { Total Surface } \\
\text { Energy, } \gamma^{\mathrm{T}} \\
\left(\mathrm{mJm}^{-2}\right)\end{array}$ & $\begin{array}{c}\text { Surface } \\
\text { Oxygen } \\
\text { Content } \\
\text { (\%at.) }\end{array}$ & $\begin{array}{c}\text { Contact } \\
\text { Angle } \\
\left({ }^{\circ}\right)\end{array}$ \\
\hline $\mathrm{AR}$ & 0.126 & 0.029 & 17.69 & 29.66 & 47.34 & 13.26 & $56.4 \pm 1.2$ \\
\hline \multicolumn{8}{|c|}{$\mathrm{CO}_{2}$ Laser-Induced Patterned Samples } \\
\hline CT50 & 0.636 & 0.148 & 12.24 & 28.63 & 40.87 & 14.33 & $66.0 \pm 4.0$ \\
\hline CT100 & 0.297 & 0.185 & 16.86 & 29.83 & 46.69 & 14.05 & $57.5 \pm 2.4$ \\
\hline $\mathrm{CH} 50$ & 0.423 & 0.103 & 10.93 & 31.64 & 42.58 & 14.99 & $65.8 \pm 2.9$ \\
\hline $\mathrm{CH} 100$ & 0.326 & 0.155 & 13.63 & 30.37 & 44.00 & 14.84 & $62.2 \pm 2.3$ \\
\hline \multicolumn{8}{|c|}{$\mathrm{CO}_{2}$ Whole Area Irradiative Processed Samples } \\
\hline CWA17 & 0.111 & 0.060 & 18.36 & 28.75 & 47.11 & 13.56 & $55.8 \pm 2.7$ \\
\hline CWA26 & 0.100 & 0.158 & 19.67 & 28.35 & 48.02 & 13.86 & $54.6 \pm 3.5$ \\
\hline CWA34 & 0.101 & 0.092 & 14.89 & 38.55 & 53.43 & 14.34 & $56.4 \pm 3.5$ \\
\hline CWA51 & 0.341 & 0.139 & 10.66 & 38.59 & 49.26 & 15.45 & $64.1 \pm 2.2$ \\
\hline CWA102 & 4.356 & 1.236 & 28.49 & 23.82 & 52.31 & 16.77 & $46.6 \pm 4.7$ \\
\hline CWA128 & 3.201 & 1.335 & 31.98 & 22.78 & 54.76 & 18.93 & $43.0 \pm 3.7$ \\
\hline
\end{tabular}


Table 2 - Results summary for samples showing roughness parameters, surface oxygen content and wettability characteristics for $\mathrm{KrF}$ processed nylon 6,6.

\begin{tabular}{|c|c|c|c|c|c|c|c|}
\hline Sample ID & $\begin{array}{l}\mathrm{Sa} \\
(\mu \mathrm{m})\end{array}$ & $\begin{array}{c}\mathrm{Ra} \\
(\mu \mathrm{m})\end{array}$ & $\begin{array}{c}\text { Polar } \\
\text { Component, } \gamma^{\mathrm{P}} \\
\left(\mathrm{mJm}^{-2}\right)\end{array}$ & $\begin{array}{c}\text { Dispersive } \\
\text { Component, } \gamma^{\mathrm{D}} \\
\left(\mathrm{mJm}^{-2}\right)\end{array}$ & $\begin{array}{c}\text { Total Surface } \\
\text { Free Energy, } \gamma^{\mathrm{T}} \\
\quad\left(\mathrm{mJm}^{-2}\right)\end{array}$ & $\begin{array}{c}\text { Surface } \\
\text { Oxygen } \\
\text { Content } \\
\text { (\%at.) }\end{array}$ & $\begin{array}{c}\text { Contact } \\
\text { Angle } \\
\left({ }^{\circ}\right)\end{array}$ \\
\hline AR & 0.126 & 0.029 & 17.69 & 29.66 & 47.34 & 13.26 & $56.4 \pm 1.2$ \\
\hline \multicolumn{8}{|c|}{ Laser-Induced Patterned Samples } \\
\hline ET50 & 0.281 & 0.312 & 15.48 & 28.74 & 44.22 & 13.87 & $60.6 \pm 3.0$ \\
\hline ET100 & 1.320 & 0.679 & 6.52 & 23.54 & 30.06 & 13.53 & $80.8 \pm 5.2$ \\
\hline EH50 & 0.739 & 0.548 & 12.94 & 17.24 & 30.17 & 14.15 & $75.1 \pm 3.9$ \\
\hline EH100 & 1.530 & 1.032 & 17.06 & 13.55 & 30.61 & 14.83 & $73.0 \pm 3.7$ \\
\hline \multicolumn{8}{|c|}{ Whole Area Irradiative Processed Samples } \\
\hline EWA100 & 0.119 & 0.029 & 21.72 & 27.08 & 48.79 & 13.11 & $52.8 \pm 1.1$ \\
\hline EWA150 & 0.092 & 0.039 & 25.98 & 24.76 & 50.74 & 13.03 & $49.1 \pm 4.7$ \\
\hline EWA200 & 0.108 & 0.030 & 25.26 & 25.86 & 51.12 & 13.28 & $48.9 \pm 2.6$ \\
\hline EWA250 & 0.107 & 0.031 & 22.37 & 28.66 & 51.03 & 13.24 & $50.3 \pm 1.5$ \\
\hline EWA250_500 & 0.164 & 0.039 & 33.31 & 21.40 & 54.71 & 15.32 & $42.9 \pm 1.6$ \\
\hline EWA250_1000 & 0.198 & 0.036 & 37.56 & 20.89 & 58.46 & 18.36 & $37.6 \pm 3.2$ \\
\hline
\end{tabular}

As given in Table 1, for all samples with the exception of whole area samples CWA17, CWA26 and CWA34 there was a significant increase in the surface roughness in comparison to the as-received sample (AR) which had a roughness of $\mathrm{Sa}, 0.1 \mu \mathrm{m}$, and $\mathrm{Ra}$, $0.03 \mu \mathrm{m}$. It should be noted that as a result of the fluences being close to that of the threshold which were used for samples CWA17, CWA26 and CWA34 the surface roughness results obtained for these samples can be seen to be equivalent to that of the asreceived sample (AR). On the other hand, it can be seen that the largest surface roughness arises from samples CWA102 and CWA128 with Sa of 4 and 3 $\mu \mathrm{m}$, respectively.

On account of the $\mathrm{KrF}$ Excimer laser whole area processing the samples had roughnesses equivalent to that of the as-received sample (AR) which had an Sa of $0.126 \mu \mathrm{m}$ and an Ra of $0.029 \mu \mathrm{m}$ (see Table 2). This can be accounted for by the observation through the WLI which showed a negligible effect on the surface topography for these samples. This further confirms that the fluences implemented for these samples was not sufficient to elicit an ablative response from the nylon 6,6 samples. Having said that, it may still have been possible for surface chemistry changes to occur This is on account of the fact that directly after the irradiation of the samples using the $\mathrm{KrF}$ excimer laser, the samples were warm to the touch, with the warmest sample being WA250_1000 which had 1000 pulses and the highest fluence of $91 \mathrm{mJcm}-2$

\section{Wettability $-\mathrm{CO}_{2}$ and $\mathrm{KrF}$ Excimer Laser-Induced Patterning}

Throughout this work it has been observed that $\mathrm{CO}_{2}$ and $\mathrm{KrF}$ excimer laser-induced patterning of nylon 6,6 inherently gives rise to a significant increase in $\theta$ (see Table 1 and Table 2). This could be seen to be on account of the likelihood of a mixed-state wetting regime taking place along the liquid-surface interface $(9,10)$. This is due to the fact that the periodic laserinduced surface would have given rise to a liquid droplet in an equilibrium state that cannot be explained through the Wenzel and Cassie-Baxter wetting regimes.

Current theory has not been corroborated with, in terms of the laser-induced patterning of nylon 6,6, insofar as the increase in $\mathrm{Sa}$ and $\mathrm{Ra}$ have not given rise to a reduction in $\theta$. However, it has been seen that surface energy parameters may have a significant impact on the wetting nature of nylon 6,6 even if a wetting transition has taken place. Figure 1 and Figure 2 show the relationship between $\theta$ and $\gamma^{\mathrm{P}}$ and $\gamma^{\mathrm{T}}$, respectively. From these graphs it is possible to identify that by collating all results for the patterning of nylon $\theta$ was a strong inverse function in correlation with $\gamma^{\mathrm{P}}$ and $\gamma^{\mathrm{T}}$. On account of this, it is reasonable to say that for the patterned nylon 6,6 samples $\gamma^{\mathrm{P}}$ and $\gamma^{\mathrm{T}}$ were dominant parameters for determining the wettability. 


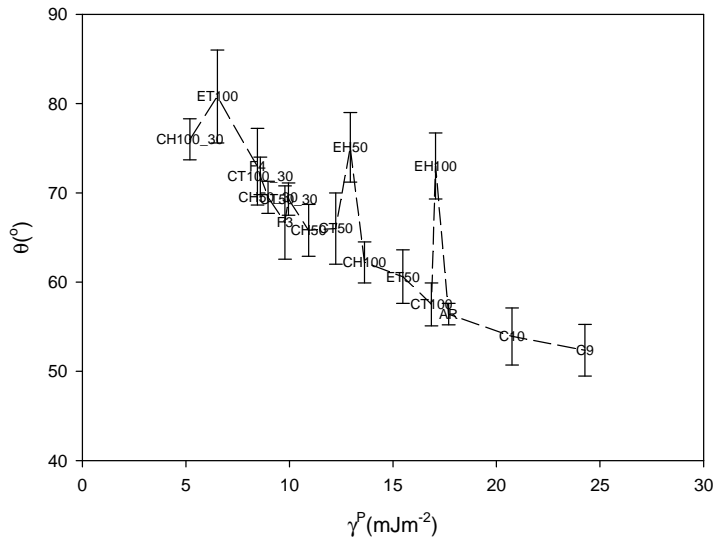

Figure $1-$ Graph showing the correlation between $\theta$ and $\gamma^{\mathrm{P}}$.

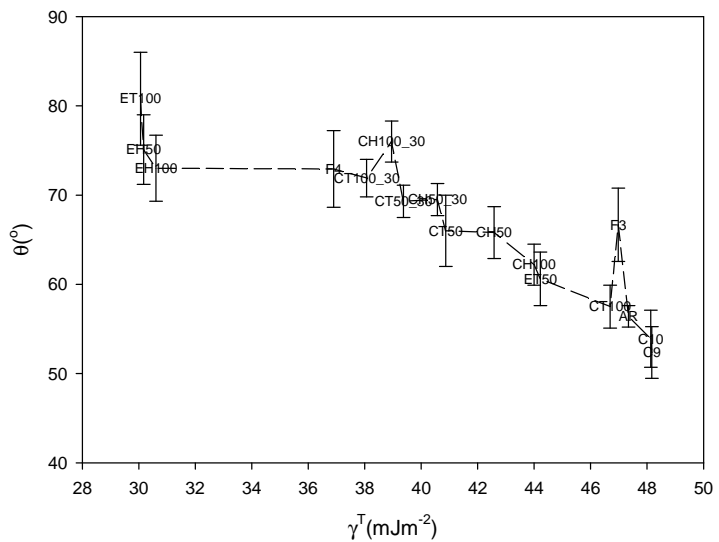

Figure 2 - Graph showing the correlation between $\theta$ and $\gamma^{\mathrm{T}}$.

Surface roughness is another surface parameter which could have a significant impact upon the way in which a material wets. However, from Figure 3 it can be seen that the surface roughness in terms of $\mathrm{Ra}$ and $\mathrm{Sa}$ did not seem to have a dominant role in determining $\theta$ for the laser-induced patterned nylon 6,6 samples. This is due to $\theta$ being slightly erratic as the surface roughness increased.

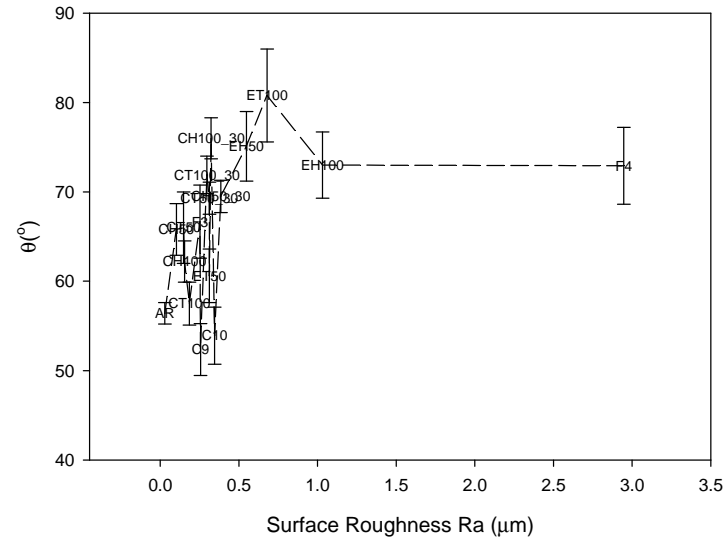

(a)

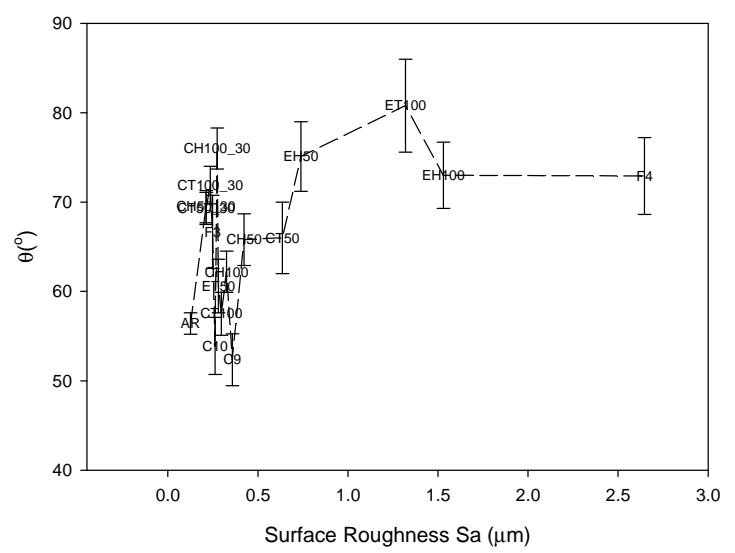

(b)

Figure 3 - Graphs showing the correlation between $\theta$, (a) $\mathrm{Ra}$ and (b) Sa.

\section{$\mathrm{CO}_{2}$ and $\mathrm{KrF}$ Excimer Laser Whole Area Irradiative Processing}

The work involving $\mathrm{CO}_{2}$ and $\mathrm{KrF}$ excimer laser whole area irradiative processing has shown that the wettability characteristics of nylon 6,6 did tend to correspond with current theory in that $\theta$ decreased on account of an increase in surface roughness, increase in $\gamma^{\mathrm{P}}$, increase in $\gamma^{\mathrm{T}}$ and increase in surface oxygen content. From Figure 4 it was found upon collating the results for all laser whole area irradiative processed nylon 6,6 samples that there was a strong inverse relationship between $\theta$ and $\gamma^{\mathrm{P}}$. In addition to this, it can also be seen from Figure 5 that $\gamma^{\mathrm{T}}$ showed a similar correlation with $\theta$. This further suggests that the surface energy parameters are the most dominant parameter in giving rise to $\theta$. What is more, Figure 4 and Figure 5 indicate that it may be possible to use $\gamma^{\mathrm{P}}$ 
and $\gamma^{T}$ to predict $\theta$ on account that they show reproducible results. That is, those samples which had similar values of either $\gamma^{\mathrm{P}}$ or $\gamma^{\mathrm{T}}$ gave rise to equivalent $\theta$.

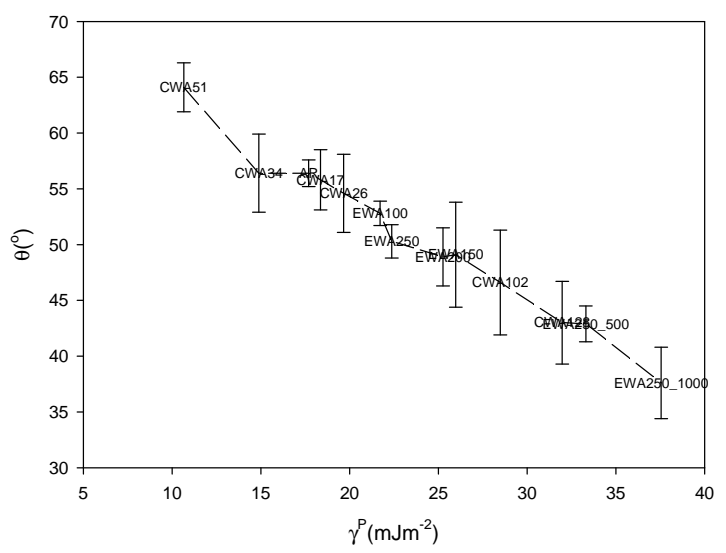

Figure 4 - Graph showing the correlation between $\theta$ and $\gamma^{\mathrm{P}}$.

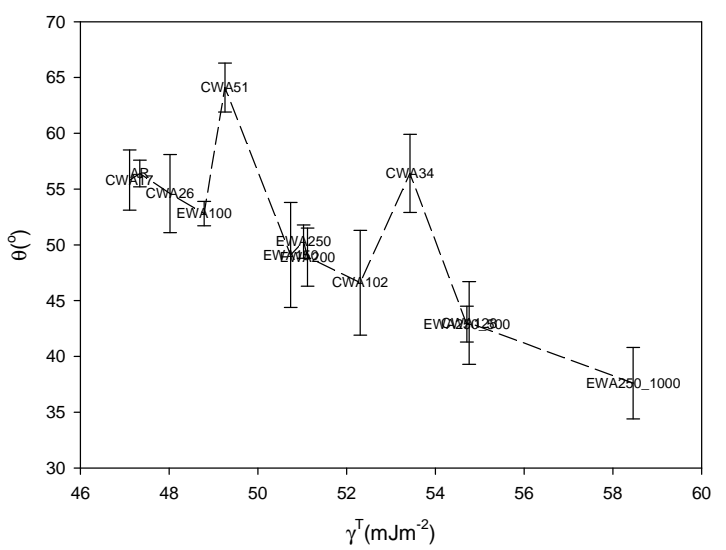

Figure 5 - Graph showing the correlation between $\theta$ and $\gamma \mathrm{T}$.
As surface roughness had increased, it appeared that Sa and $\mathrm{Ra}$ may have a significant impact upon $\theta$; however, it can be seen from Figure 6 that the surface roughness did not tend to have any particular correlation with $\theta$. This is highly noteworthy as it allows one to further deduce that from the results of the laser whole area processed nylon 6,6 samples $\gamma^{\mathrm{P}}$ and $\gamma^{\mathrm{T}}$ were the most dominant parameter in determining $\theta$.

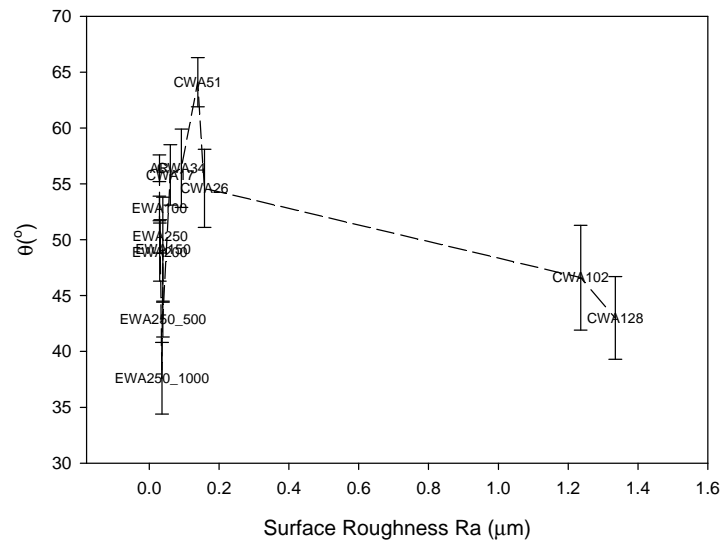

(a)

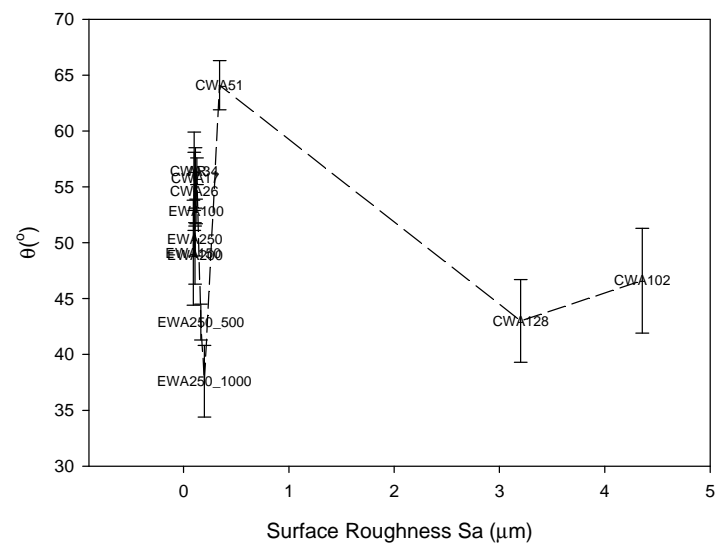

(b)

Figure 6 - Graphs showing the correlation between $\theta$, (a) Ra and (b) Sa.

\section{Comparisons Between Laser-Induced Patterning and Laser Whole Area Irradiative Processing}

For all $\mathrm{CO}_{2}$ and $\mathrm{KrF}$ excimer laser-induced patterning of nylon 6,6 the surface oxygen content had increased which can be attributed to oxidation of the surface during the laser processing. It is reasonable to conclude 
from the results shown in Table 1 and Table 2 that surface oxygen content in this instance was not a main driving parameter which accounts for the measured $\theta$. This is owed to variations in $\theta$ between the laserinduced patterned and laser whole area irradiative processed samples.

When compared to one another it was found that all laser-induced surface patterning elicited an increase in $\theta$; whereas the laser whole area irraditative processing of the nylon 6,6 gave rise to a more hydrophilic surface in which $\theta$ decreased. In order to explain the increase in $\theta$ for the laser-induced patterned samples a mixedstate wetting regime is proposed in which both CassieBaxter and Wenzel regimes occur along the interface once the droplet is in equilibrium. Thus the mixed-state wetting regime is believed to arise from the rough periodic patterns induced by the laser processing, allowing one to infer that the periodic topographical patterns have a dominant role in the determination of $\theta$. Having said that, upon collating the results from all studied samples it is possible to see from Figure 7 that $\gamma^{\mathrm{P}}$ is highly correlated with $\theta$, such that an increase in $\gamma^{\mathrm{P}}$ gave rise to a reduction in $\theta$ regardless of the topography or surface modification technique implemented. In addition to this, Figure 8 shows that $\theta$ was also an inverse function of $\gamma^{\mathrm{T}}$ in a similar manner to that seen with $\gamma^{\mathrm{P}}$. This is highly significant as this shows that the surface energy parameters are one of the most dominant parameters in determining the wetting nature of laser-modified nylon 6,6.

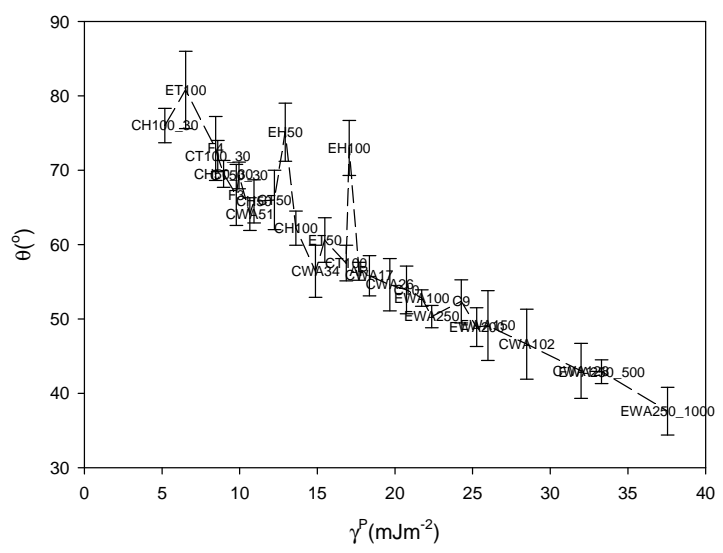

Figure 7 - Graph showing the correlation between $\theta$ and $\gamma^{\mathrm{P}}$.

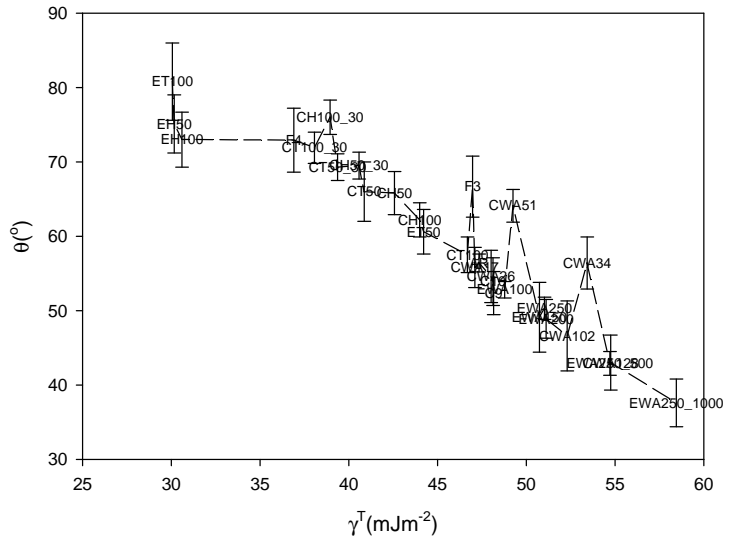

Figure 8 - Graph showing the correlation between $\theta$ and $\gamma^{\mathrm{T}}$.

In order to confirm that the surface energy parameters were the most dominant in terms of the wettability characteristics of nylon 6,6, Figure 9 shows that surface roughness in regards to $\mathrm{Ra}$ and $\mathrm{Sa}$ did not have a good correlation with $\theta$. This could be on account of these results including both laser-induced patterning and laser whole area irradiative processing results in which a transition in wetting regime has been proposed to account for the differences in measured $\theta$. As a result of this, it can be said that from the evidence presented here the surface energy parameters; namely $\gamma^{\mathrm{P}}$ and $\gamma^{\mathrm{T}}$ are the central factor which govern the wetting nature of nylon 6,6 , irrespective of the surface modification used or arising wetting regime. 


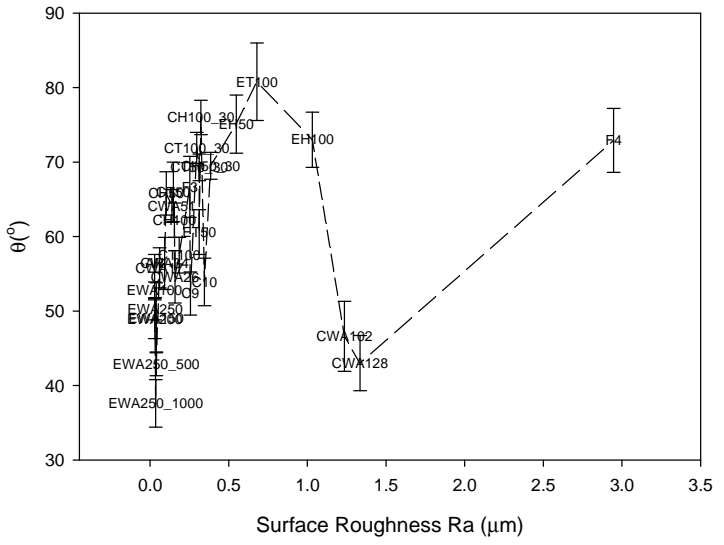

(a)

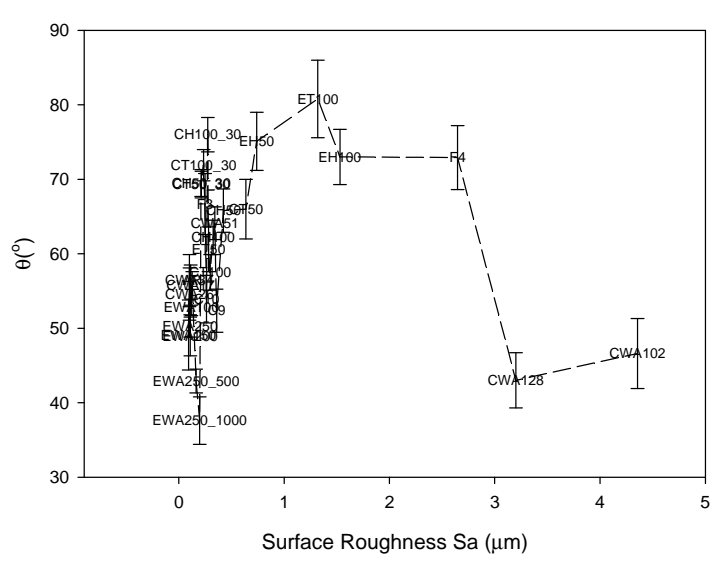

(b)

Figure 9 - Graphs showing the correlation between $\theta$, (a) Ra and (b) Sa.

\section{Conclusions}

By implementing two different laser types, namely $\mathrm{CO} 2$ and $\mathrm{KrF}$ excimer lasers, it has been determined that both the surface of nylon 6,6 can be greatly modified. All of the laser processing techniques implemented, with the exception of the $\mathrm{KrF}$ excimer laser whole area irradiative processing, lead to a significant increase in $\mathrm{Ra}$ and $\mathrm{Sa}$ for the nylon 6,6 samples. The exception of the $\mathrm{KrF}$ excimer laser whole area irradiative process samples can be explained by the fact that most of the samples had an incident fluence below that of the threshold fluence. Then, for those samples with incident fluences just above the threshold only limited melting would have taken place due to the fluences being relatively small, especially when compared to the fluence used for the laserinduced patterning.
As a result of the surface modifications that had taken place it was also seen that the surface energy, $\gamma^{\mathrm{T}}$, and its components, namely $\gamma^{\mathrm{P}}$, had been modulated to a great extent. This, along with the other surface modifications that had taken place gives further evidence as to the attractive means of using laser technology to manipulate both the surface topography and surface chemistry of nylon 6,6 simultaneously. What is more, it is highly likely that other polymeric materials could be modified in a similar manner and that laser technology could very well be applied to a number of applications which requires the surface modification of polymers.

With the effects on the surface topography and surface chemistry being observed it was further seen that these surface modifications had a large impact upon the wettability of the nylon 6,6. It was found that there was differing resulting $\theta$ for the two different processing techniques implemented; laser-induced patterning and laser whole area irradiative processing. That is, for the laser-induced patterned samples there was a significant increase in $\theta$ compared to the as-received sample which can be attributed to a mixed-state wetting regime arising from the rough periodic laser-induced pattern.

It has also been determined that there was a very strong relationship between $\theta, \gamma^{\mathrm{P}}$ and $\gamma^{\mathrm{T}}$. Since, $\theta$ was found to be a strong correlative decreasing function of both $\gamma^{\mathrm{P}}$ and $\gamma^{\mathrm{T}}$ which identified that the surface energy played a major role in the determination of the wettability of nylon 6,6. With this in mind, it is reasonable to say that this result allows one to realize that the wettability of laser-modified nylon 6,6 could be predicted by knowing the surface energy and its components.

As a result of the laser surface treatment and arising surface modifications it has been seen that the wettability characteristics of nylon 6,6 can be significantly modulated. Leading on form this, it has also been determined that a number of parameters, namely $\gamma \mathrm{P}$ and $\gamma \mathrm{T}$, can be implemented to predict the wettability nature of laser surface treated nylon 6,6. With this in mind, it has been further evidenced that lasers offer an attractive means to surface treat nylon 6,6 in order to modulate the wettability characteristics.

\section{References}

(1) Adamson AW. (1973) Potential Distortion Model for Contact Angle and Spreading II. Temperature Dependant Effects. J. Coll. Inter. Sci. 44 273-281. 
(2) Bauerle D. Laser Processing and Chemistry. Third ed. New York, USA: Springer-Verlag; 2000.

(3) Nebe B, Luthen F, Lange R, Becker P, Beck U, Rychly J. (2004) Topography-induced alterations in adhesion structures affect mineralization in human osteoblasts on titanium. Mater. Sci. Eng. C 24 619624.

(4) Karaca E, Hockenbeger A.S. (2008) Analysis of the Fracture Morphology of Polyamide, Polyester, Polypropylene, and Silk Sutures Before and After Implantation In Vivo. J. Biomed. Mater. Res. B: Appl. Biomat. 87 580-589.

(5) Waugh DG, Lawrence J, Morgan DJ, Thomas CL. (2009) Interaction of $\mathrm{CO}_{2}$ laser-modified nylon with osteoblast cells in relation to wettability. Mater. Sci. Eng. C 29 2514-2524.

(6) Waugh DG, Lawrence J. (2011) The enhancement of biomimetic apatite coatings by means of $\mathrm{KrF}$ excimer laser surface treatment of nylon 6,6. Lasers Eng. 21 95-114

(7) Waugh DG, Lawrence J. (2010) On the use of CO2 laser induced patterns to modify the wettability of poly(methyl methamecrylate) (PMMA). Opt. Lasers Eng. 48 707-715.

(8) Renke-Gluszko M, Fray ME. (2004) The effect of simulated body fluid on the mechanical properties of multiblock poly(aliphatic/aromatic-ester) copolymers. Biomaterials 25 5191-5198.

(9) Chen X, Lu T. (2009) The apparent state of droplets on a rough surface. Sci. China Ser. G. Phys. Mech. Astron. 52 233-238.

(10) Wu X, Zheng L, Wu D. (2005) Fabrication of superhydrophobic surfaces from microstructured $\mathrm{ZnO}$ based surfaces via a wet-chemical route. Langmuir 21 2665-2667.

\section{Meet the Author}

Dr. David Waugh completed his Ph.D. under the supervision of Dr. Jonathan Lawrence in July 2010. Dr. Waugh is now a Lecturer at the newly established School of Engineering at the University of Lincoln, UK. He is also part of the Lincoln Laser Engineering Group in which he primarily focuses on the application of laser surface treatment to life sciences. He obtained his MPhys Hons. in Physics with Lasers and Photonics and MSc in Laser Applications in Micro-Machining and Processing from the University of Hull, UK. 